\title{
Temporal Stability of Block Term Decomposition in Noninvasive Atrial Fibrillation Analysis
}

\author{
Pedro Marinho R. de Oliveira and Vicente Zarzoso \\ Université Côte d'Azur, CNRS, I3S Laboratory \\ CS 40121, 06903 Sophia Antipolis Cedex, France \\ Email: \{marinho, zarzoso\}@i3s.unice.fr
}

\begin{abstract}
Considered as the last great frontier of cardiac electrophysiology, atrial fibrillation (AF) is the most common sustained cardiac arrhythmia in clinical practice. The mechanisms behind this disease are not completely understood, and a precise analysis of the atrial activity (AA) signal is necessary to a better understanding. The block term decomposition (BTD) has been recently proposed as a useful tool for noninvasive AA extraction in electrocardiogram (ECG) signals. However, this tensor factorization technique was assessed only in short fixed segments of an AF ECG. To bridge this gap, the present work evaluates for the first time the temporal (intra-patient) stability of BTD by considering consecutive segments through a whole AF ECG recording. Experimental results show the consistency of BTD as an AA extraction tool, being capable of outperforming two well-known matrix-based methods and presenting a stable performance over time.
\end{abstract}

\section{INTRODUCTION}

Atrial fibrillation (AF) is the most common sustained cardiac arrhythmia encountered in clinical practice and is considered a major health and economical concern, since about 160000 new patients are diagnosed with AF every year in USA only, and similar numbers are shown in some European countries [1]. Also, a patient with AF costs anually approximately $\$ 8700$ more in healthcare than a patient without AF. The treatment of this disease is estimated to add $\$ 26$ billion per year to the USA healthcare bill [2]. The importance given to this supraventricular arrhythmia has been increased in the past few years and is expected to increase even more, since its mechanisms are not yet completely understood. This makes AF a challenging cardiac condition, considered as the last great frontier of cardiac electrophysiology. During AF, the $\mathrm{P}$ wave, that corresponds to a normal atrial activation, is replaced by f waves (fibrillatory waves), which are present through all the electrocardiogram (ECG) recording, but masked by the QRST complex that corresponds to the ventricular activity (VA) in each heartbeat.

To better understand the mechanisms behind $\mathrm{AF}$, it is necessary to extract the atrial activity (AA) from the ECG for an accurate analysis of the $f$ waves. A noninvasive method to extract the AA from the cardiac signals recorded by the standard 12-lead ECG is using matrix decompositions techniques to solve blind source separation (BSS) problems, such as principal component analysis (PCA) and independent component analysis (ICA) [3]-[5]. Matrix decomposition techniques have proved to be useful in AA extraction. However, it is known that they have some limitations since constraints need to be imposed to guarantee the uniqueness of such decompositions. Although mathematically coherent, such constraints may lack physiological grounds, making difficult the results interpretation.

To overcome the limitations of matrix decompositions, a tensor approach has been recently proposed to extract AA in AF ECGs [6]-[9]. Compared to matrix-based techniques, tensor decompositions present some remarkable features such as essential uniqueness with reduced or minimum constraints and the fact that the rank of the tensor can exceed its dimensions, whereas in matrices the rank is limited by the matrix lowest dimension. The block term decomposition (BTD) proposed in [14] suits the characteristics of AA in an AF signal, since atrial signals can be approximated by all-pole (exponential) models and mapped onto Hankel matrices with rank equal to the number of poles [9]. The Hankel matrices containing the ECG data are stacked in the third dimension of a $3^{\text {rd }}$-order tensor, and then processed by BTD. Previous experimental results in synthetic and real ECG data showed that BTD can outperform the matrix-based techniques for AA extraction in short [6]-[10] and long [11] segments of AF ECG recordings.

The BTD has proved to be useful extracting the AA signal from the AF ECG, being able to outperform the matrixbased techniques. However, this tensorial approach was only assessed in short segments. Its performance in a whole ECG remains an open question. To answer this question and provide results about the temporal stability of this new promising technique, the present work assesses BTD for the first time in a whole ECG of a patient with persistent AF and compares it to two popular matrix-based methods for AA extraction: RobustICA-f [12] and PCA [13]. Experimental results using Monte Carlo runs evaluate the intra-patient stability of BTD regarding the AA extraction from AF ECGs, showing the consistency of this technique.

The rest of this paper is organized as follows. In Section II, the notation used in the work is introduced. Section III recalls the BTD as a tensor approach to solve BSS problems and discusses quantitative measures of AA content. Section IV reports the experimental results and, finally, Section V presents the conclusion of this work and the perspectives of future works. 


\section{NOTATIONS}

Scalars, vectors, matrices and tensors are represented by lower-case $(a, b, c, \ldots)$, boldface lower-case $(\mathbf{a}, \mathbf{b}, \mathbf{c}, \ldots)$, boldface capital $(\mathbf{A}, \mathbf{B}, \mathbf{C}, \ldots)$ and calligraphic $(\mathcal{A}, \mathcal{B}, \mathcal{C}, \ldots)$ letters, respectively.

The transpose is represented by $(\cdot)^{T}$, symbol $\|\cdot\|$ represents the $l_{2}$-norm and $\circ$ represents the outer product. The operator $\operatorname{diag}(\cdot)$ builds a diagonal matrix by placing its arguments along the diagonal. Given a $3^{\text {rd }}$-order tensor $\mathcal{A} \in \mathbb{C}^{I_{1} \times I_{2} \times I_{3}}$, with entries $a_{i_{1}, i_{2}, i_{3}}$, its frontal slices are represented by $\mathbf{A}_{. . i_{3}} \in \mathbb{C}^{I_{1} \times I_{2}}$. Given a matrix $\mathbf{A} \in \mathbb{C}^{I_{1} \times I_{2}}$, with entries $a_{i_{1}, i_{2}}$, its $i_{1}^{\text {th }}$ row and $i_{2}^{\text {th }}$ column are represented by $\mathbf{a}_{i_{1}}$. and $\mathbf{a}_{. i_{2}}$, respectively.

\section{MethodS}

\section{A. Block Term Decomposition}

The BTD of an arbitrary third-order tensor $\mathcal{T} \in \mathbb{R}^{I_{1} \times I_{2} \times I_{3}}$ is written as:

$$
\mathcal{T}=\sum_{r=1}^{R} \mathbf{E}_{r} \circ \mathbf{c}_{r}
$$

where $\mathbf{c}_{r} \in \mathbb{R}^{I_{3}}$ and $\mathbf{E}_{r} \in \mathbb{R}^{I_{1} \times I_{2}}$ has rank $L_{r}$ and admits a decomposition $\mathbf{E}_{r}=\mathbf{A}_{r} \mathbf{B}_{r}^{T}$, where $\mathbf{A}_{r} \in \mathbb{R}^{I_{1} \times L_{r}}$ and $\mathbf{B}_{r} \in$ $\mathbb{R}^{I_{2} \times L_{r}}$ have rank $L_{r}$. Equation (1) can be rewritten as:

$$
\mathcal{T}=\sum_{r=1}^{R}\left(\mathbf{A}_{r} \mathbf{B}_{r}^{T}\right) \circ \mathbf{c}_{r} .
$$

If the matrix factors $\mathbf{A}=\left[\begin{array}{llll}\mathbf{A}_{1} & \mathbf{A}_{2} & \ldots & \mathbf{A}_{R}\end{array}\right] \in$ $\mathbb{R}^{I_{1} \times \sum_{r=1}^{R} L_{r}}$ and $\mathbf{B}=\left[\begin{array}{llll}\mathbf{B}_{1} & \mathbf{B}_{2} & \ldots & \mathbf{B}_{R}\end{array}\right] \in \mathbb{R}^{I_{2} \times \sum_{r=1}^{R} L_{r}}$ are full-column rank, which requires that $\sum_{r=1}^{R} L_{r} \leq I_{1}, I_{2}$, and $\mathbf{C}=\left[\begin{array}{llll}\mathbf{c}_{1} & \mathbf{c}_{2} & \ldots & \mathbf{c}_{R}\end{array}\right] \in \mathbb{R}^{I_{3} \times R}$ does not contain proportional columns, then the BTD is essentially unique [14, Theorem 2.2].

The ECG data matrix, with $K$ leads and $N$ samples, can be modeled as:

$$
\mathbf{Y}=\mathbf{M S} \in \mathbb{R}^{K \times N}
$$

where $\mathbf{M} \in \mathbb{R}^{K \times R}$ is the mixing matrix, modeling the propagation of the cardiac electrical sources from the heart to the body surface, $\mathbf{S} \in \mathbb{R}^{R \times N}$ is the source matrix that contains the atrial, ventricular and noise sources, and $R$ is the number of sources [5]. The AA extraction in an AF ECG recording can be seen as a BSS problem, since the only data observed is the matrix $\mathbf{Y}$, from which matrices $\mathbf{M}$ and $\mathbf{S}$ are to be estimated. In [14], the BTD is proposed as a solution of a BSS problem like (3). The idea to obtain a tensor from $\mathbf{Y}$ is to map its $k^{\text {th }}$ row onto a Hankel matrix and then stack each Hankel matrix along the third dimension (as frontal slices) of a third-order tensor $\mathcal{Y} \in \mathbb{R}^{I \times J \times K}$, where $I=J=\frac{N+1}{2}$ if $N$ is odd or $I=\frac{N}{2}$ and $J=\frac{N}{2}+1$ if $N$ is even. This way, the tensor $\mathcal{Y}$ can be written as:

$$
\mathcal{Y}=\sum_{r=1}^{R} \mathbf{H}_{\mathbf{S}}^{(r)} \circ \mathbf{m}_{\cdot r} .
$$

Equation (4) shows that the tensor ECG data follows a BTD tensor model. During AF, atrial sources can be represented by the exponential (or all-pole) model as:

$$
s_{r, n}=\sum_{\ell=1}^{L_{r}} \lambda_{\ell, r} z_{\ell, r}^{n-1}
$$

where $n=1, \ldots, N, r=1, . ., R, L_{r}$ is the number of exponential terms, $z_{\ell, r}$ is the $\ell^{\text {th }}$ pole of the $r^{\text {th }}$ source, and $\lambda_{\ell, r}$ is the corresponding scaling coefficient. This way, their associated Hankel matrix accepts the Vandermonde decomposition as in [15].

In the case of different poles, the Vandermonde matrix with $L_{r} \leq I, J$ will have full-column rank $L_{r}$, so if $\mathbf{M}$ does not have proportional columns, the BTD in (4) will be essentially unique. In the case of equal poles, milder conditions can assure the uniqueness of (4) [14].

\section{B. Atrial Activity Content Measurement}

Signal processing methods used to solve BSS problems separate the original signal in several sources. In AF ECG, typically at least one of these sources contains the AA. However, it is unknown if the AA is concentrated only in a single source. The present work, as previous ones, considers that the AA is concentrated in a single source, and the source with the most AA content is called the atrial source. A sucessful AA extraction depends on the accurate selection of the atrial signal among the others estimated sources. In [10] the BTD showed that the classical method of atrial source selection [3], [4] does not provide a satisfactory accuracy, and then two sub-optimal methods that outperform the classical one were proposed. In this work the atrial source is selected using the method proposed in [10] that provided the highest accuracy.

In the frequency domain, the AA during $\mathrm{AF}$ has a peak between 3 and $9 \mathrm{~Hz}$. The position of this peak is called dominant frequency (DF). We define as potential atrial sources any source with DF placed between such interval. To evaluate the AA extraction performance, three parameters are used to measure its quality. The first parameter is the spectral concentration (SC), that is, the relative amount of energy around the DF. The SC is computed as:

$$
S C=\frac{\sum_{0.82 f_{p}}^{1.17 f_{p}} P_{A A}\left(f_{i}\right)}{\sum_{0}^{F_{s} / 2} P_{A A}\left(f_{i}\right)}
$$

where $f_{p}$ is the value of the DF, $F_{s}$ is the sampling frequency and $P_{A A}$ is the power spectrum of the AA signal, estimated as in [4]. The second parameter used to better analyze the potential atrial sources is the kurtosis, denoted $K$, of the signal in the frequency domain acquired by a 4096-point FFT. As in [12], the general expression of kurtosis is used, valid for noncircular complex data:

$$
K=\frac{E\left[\left|\mathbf{s}_{r .}\right|^{4}\right]-2 E\left[\left|\mathbf{s}_{r}\right|^{2}\right]^{2}-\left|E\left[\mathbf{s}_{r .}^{2}\right]\right|^{2}}{E\left[\left|\mathbf{s}_{r .}\right|^{2}\right]^{2}} .
$$


Kurtosis is a measure of peakedness and sparsity of a distribution. When computed in the frequency domain, it provides a quantitative measure of harmonicity of the signal. Indeed, a high kurtosis in the frequency domain means that the power spectral density is sparse, which is suggestive of a harmonic signal like AA in AF. Also, kurtosis is parameter free, not depending on the DF or the definition of a suitable interval for interpretation, as in SC [10]. The third parameter, which is used to eliminate weak sources, is the power contribution to lead V1 given by:

$$
P(r)=\frac{1}{N}\left\|m_{r}^{(V 1)} \mathbf{s}_{r .}\right\|^{2} \quad\left(\mathrm{mV}^{2}\right)
$$

where $m_{r}^{(V 1)}$ is the contribution of the $r^{\text {th }}$ source to lead V1, given by the corresponding element of matrix $\mathbf{M}$ in Equation (3), and $\mathbf{s}_{r}$. is the $r^{\text {th }}$ source, corresponding to the $r^{\text {th }}$ row of matrix $\mathbf{S}$, also in Equation (3). The power contribution to lead V1 by an AA source is expected to be strong, since this lead is the one that typically best reflects AA in AF ECGs.

\section{EXPERIMENTAL RESULTS}

\section{A. Real AF ECG Data and Preprocessing}

The reported experiments consider 56 segments of a real standard 12-lead AF ECG recording from a patient suffering from persistent AF. These segments compose the whole ECG recording, that belongs to a database provided by the Cardiology Department of the Princess Grace Hospital Center, Monaco. The recording are acquired at a $977 \mathrm{~Hz}$ sampling rate and are preprocessed by a zero-phase forward-backward type-II Chebyshev bandpass filter with cutoff frequencies of 0.5 and $40 \mathrm{~Hz}$, in order to suppress high-frequency noise and baseline wandering.

The first three segments in lead II from the patient is shown on Figure 1. All the segments have a fixed length of 1500 samples (about 1.53 seconds) and all the 12 leads are used in the experiments. The segments are downsampled by a factor of four, resulting in $3^{\text {rd }}$-order tensors of dimensions $188 \times 188 \times$ 12 , since the originally built $3^{\text {rd }}$-order tensors of dimensions $750 \times 751 \times 12$ pose some difficulties to Tensorlab.

\section{B. BTD Setup}

The BTD is implemented using the non-linear least squares (NLS) method available in Tensorlab MATLAB toolbox [16] choosing $R=12$ and $L_{r}=L$, for $r=1,2, \ldots, R$, with $L$ taking values in the set $\{17,48,95\}$. This choice is chosen based on the work [9], that showed that such values provided good results for the heartbeat with the largest TQ segment of the patient considered in the present work. The tolerance threshold for BTD convergence is set to $10^{-9}$ and the maximum number of iterations is set to 1000. Monte Carlo runs with Gaussian random initialization for the spacial and temporal factors at each run are used to analyze the performance of BTD in each segment regarding the AA signal extraction. Monte Carlo runs are needed since the performance of BTD depends strongly on the initialization of its factors and a suitable initialization is still an open challenge.

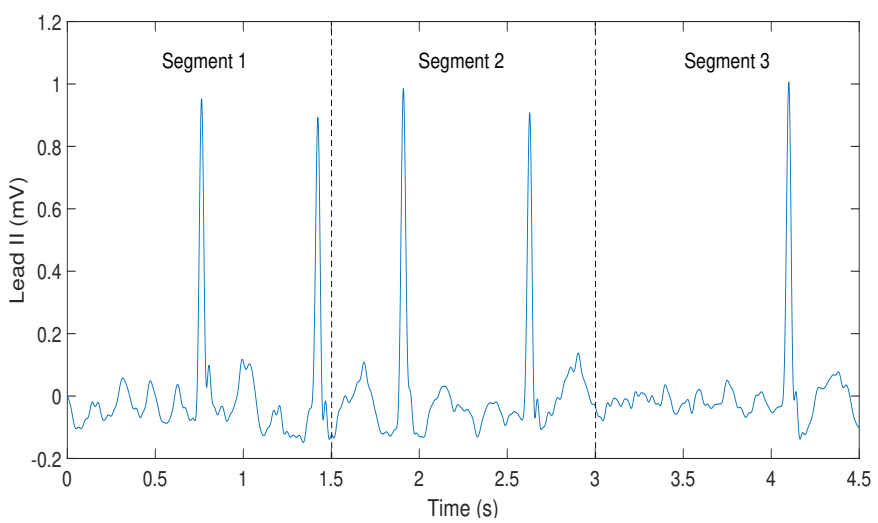

Fig. 1: The first 3 segments of the AF ECG recording composed of 56 segments. Although all the 12 leads are processed, only lead II is shown for clarity.

\section{Experiments}

Three experiments in the whole AF ECG recording are performed:

- BTD-1: A single Monte Carlo run for each segment using $L_{r}=48$.

- BTD-2: 10 Monte Carlo runs for each segment using $L_{r}=48$, then the best performance out of the 10 independent runs is chosen, considering the quality of the estimation measured by the parameters introduced in the previous section.

- BTD-3: For the segments with unsatisfactory AA extraction, i.e., with low values of $\mathrm{SC}$ and $K$, in the second experiment, 10 new Monte Carlo runs are performed changing the rank $L_{r}$ to 17 or 95 (the one which performed best), then the best performance out of the 10 Monte Carlo runs is chosen, as in the second experiment.

\section{Intra-Patient Stability}

In Figures 2 and 4, the bluebox, the redline, the whiskers and the red dots represent the 25 th and 75 th percentiles, the median, the extreme values and the outliers, respectively, of the observed data.

Figure 2 shows how the SC of the atrial source is distributed over the 56 segments that compose the whole AF ECG for the three experiments performed with BTD, as well as PCA and RobustICA-f. The improvement of BTD-2 over BTD-1 is expected and illustrates the dependence NLS method on the initialization of the matrix factors, since the model parameters are kept fixed and only more Monte Carlo runs are performed. The improvement of BTD-3 over the previous experiments illustrates the need for choosing the right model parameters in order to have satisfactory performance. This improvement is also expected, since this experiment tries to adapt the multilinear rank to each segment, using the same number of Monte Carlo runs as BTD-2. Figure 2 also shows that if the right initialization and model parameters are chosen, BTD can clearly outperform PCA and RobustICA-f, two well-know 


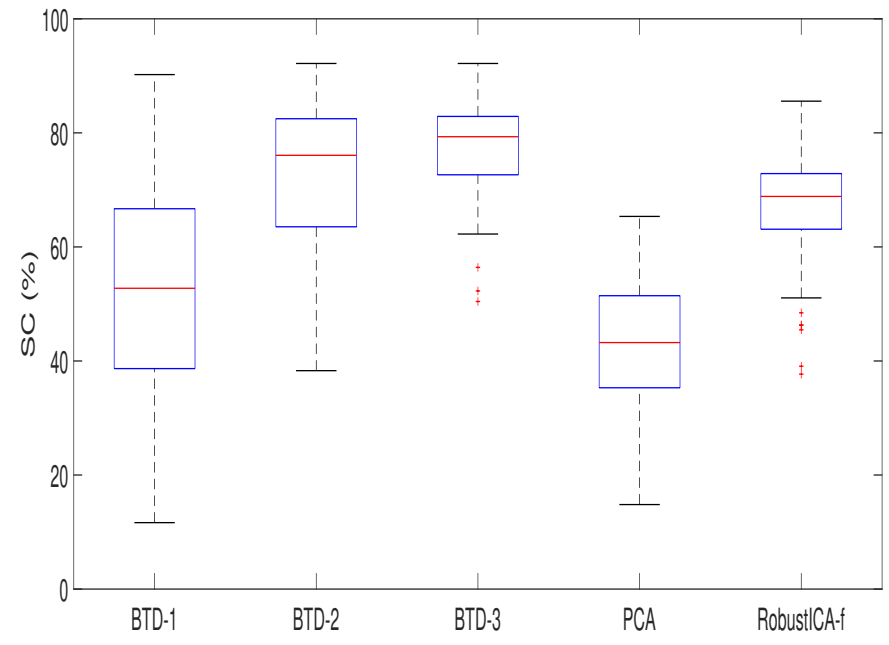

Fig. 2: Distribution of SC values (\%) for each version of BTD, as well as PCA and RobustICA-f.

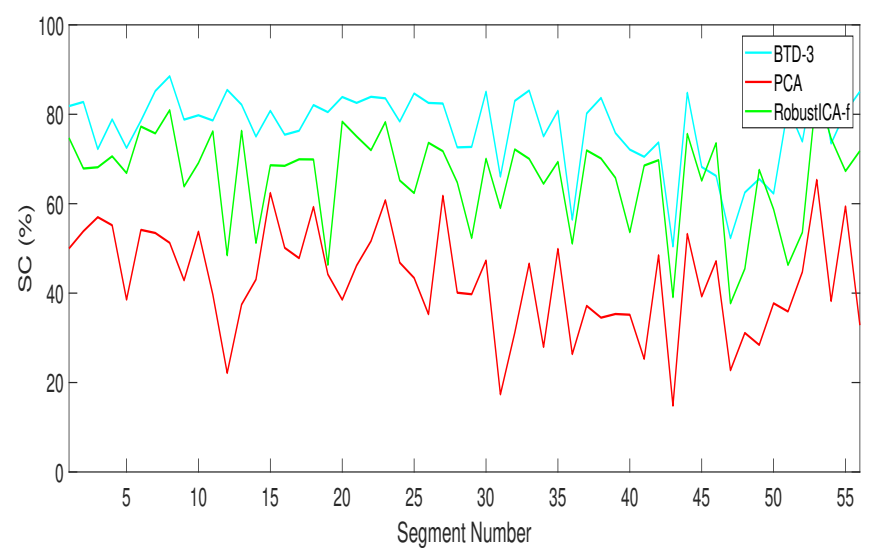

Fig. 3: Variation of SC values (\%) over the 56 segments for BTD-3, PCA and RobustICA-f.

matrix-based methods. These improvements can be viewed as the SC becomes more concentrated in high values, which means more stability.

In Figure 3, the temporal stability of BTD can be seen from another perspective, showing how the SC values of the AA signal varies over the 56 segments. For the sake of clarity, only BTD-3, the tensor version that provided the best performance, PCA and RobustICA-f are shown. It can be seen that the matrix-based methods present considerably variations over the whole ECG, whereas BTD-3 provides a SC more concentrated in high values, showing that this technique can be stable over time with a satisfactory AA extraction performance.

The superiority of BTD as an AA extraction tool over the matrix-based techniques is also shown in Table I. It can be seen that as the initialization of the factors and the model parameters are adapted to each segment, the average AA extraction quality is improved, as measured by $\mathrm{SC}$ and $K$. Since $P(r)$ is a parameter used only to eliminate weak sources and not to measure the quality of the AA extraction, it is not
TABLE I: Mean values of SC (\%) and $K$ for the three versions of BTD, as well as PCA and RobustICA-f.

\begin{tabular}{|l|c|c|}
\hline & SC & K \\
\hline BTD-1 & 53.02 & 151.33 \\
\hline BTD-2 & 71.80 & 242.35 \\
\hline BTD-3 & 76.95 & 260.41 \\
\hline PCA & 42.75 & 95.72 \\
\hline RobustICA-f & 66.10 & 216.06 \\
\hline
\end{tabular}

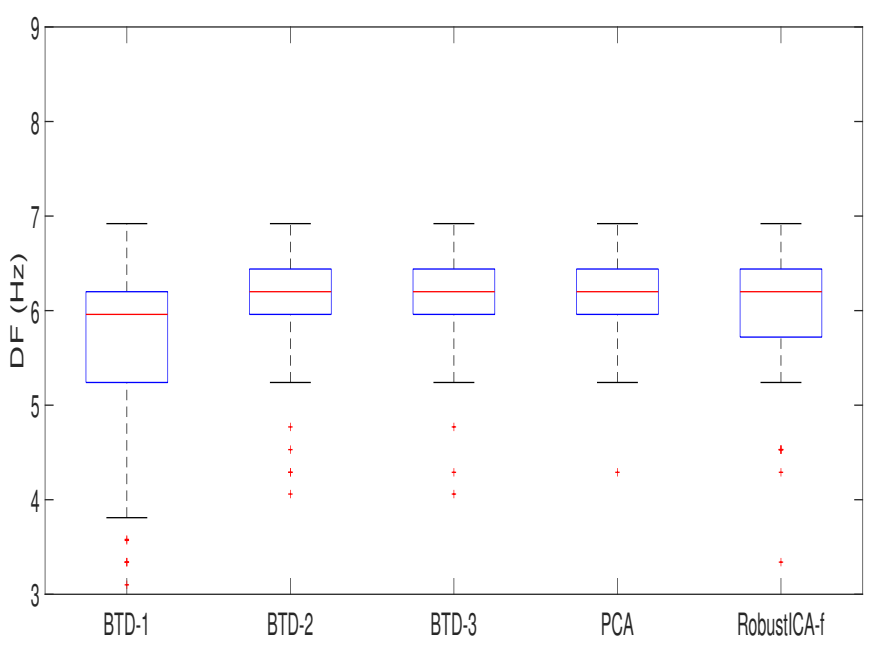

Fig. 4: Variation of DF $(\mathrm{Hz})$ for each version of BTD, as well as PCA and RobustICA-f.

shown in this table.

Since the AA signals in AF ECGs are non-stationary, it is expected that the DF slowly changes with time, i.e., over the segments of the same recording. In Figure 4 it can be seen that the DF of the estimated atrial source by BTD-2, BTD-3, PCA and RobustICA-f lies in the interval 5.24-6.67 Hz, despite the outliers. In BTD-1 the DF interval is expected to be greater, since for many of the segments this method did not provide a satisfactory AA extraction, as we used fixed parameters to compute the BTD with a single initialization of its factors.

Indeed, BTD-1 provided an unsatisfactory AA extraction in 21 out of the 56 segments. For BTD-2 this number dropped to 5, while for BTD-3 no unsatisfactory AA extractions were performed. It is fair to report that PCA performed 9 unsatisfactory AA extractions and RobustICA-f only 7. In Figure 5 it can be seen that some estimated atrial sources for PCA, BTD-1 and BTD-2 have a $P(r)$ value under $10^{-4} \mathrm{mV}^{2}$. This characterizes an unsatisfactory AA extraction, since a source with power contribution to lead V1 under this threshold does not present significant AA content, as reported in [10].

\section{CONClusion}

BTD has been recently proposed as an useful tool to atrial signal extraction in AF ECGs, showing satisfactory performances in fixed segments. The contribution of the present work is the analysis of the intra-patient variability of BTD. Three experiments show that the performance of this technique 


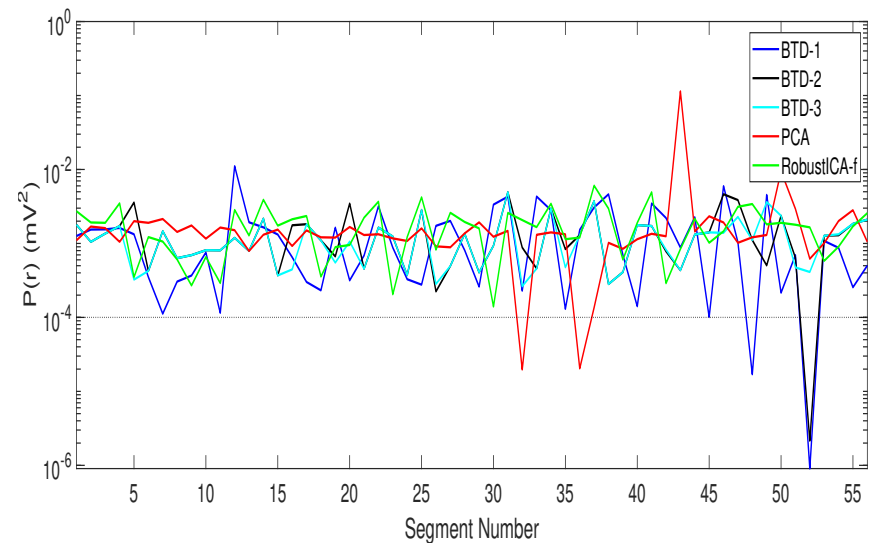

Fig. 5: Power contribution to lead V1 values $\left(\mathrm{mV}^{2}\right)$ for the selected atrial source over the 56 segments for each version of BTD, as well as PCA and RobustICA-f.

presents a strong dependence on its initialization and model parameters in this particular biomedical application. Nevertheless, with a suitable choice of its initialization and model parameters, BTD can provide not only good temporal stability, but a better estimation of the atrial source (as measured by SC and $K$ ) than two well-known matrix-based techniques. A method for computing BTD more robust to initialization is still an open challenge, as well as the optimal estimation of the model parameters for each AF ECG segment.

Taking this into account, future works would aim at developing a method of compute BTD with reduced dependence on initialization, as well as an automated method that can optimally estimate the model parameters for each particular observed segment. Increase the number of observed patients is also a prospect of future works, in order to analyze the inter-patient variability of this promising tensor technique.

\section{ACKNOWLEDGMENT}

Pedro Marinho R. de Oliveira is funded by a PhD scholarship from the IT Doctoral School of the Universite Côte d'Azur. Vicente Zarzoso is a member of the Institut Universitaire de France.

\section{REFERENCES}

[1] L. Mainardi, L. Sörnmo, S. Cerutti, "Understanding Atrial Fibrilation: The Signal Processing Contribution", Synthesis Lectures on Biomedical Engineering, Morgan \& Claypool Publishers, 2008.

[2] C. T. January, L. S. Wann, J. S. Alpert, H. Calkins, J. C. Cleveland, J. E. Cigarroa, J. B. Conti, et al., "2014 AHA/ACC/HRS guideline for the management of patients with atrial fibrillation: A report of the American College of Cardiology/American Heart Association Task Force on practice guidelines and the Heart Rhythm Society", Circulation, vol. 64, no. 21, pp. 2246-2280, Dec. 2014.

[3] J. J. Rieta, F. Castells, C. Sánchez, V. Zarzoso, and J. Millet, "Atrial activity extraction for atrial fibrillation analysis using blind source separation", IEEE Transactions on Biomedical Engineering, vol. 51, no. 7, pp. 1176-1186, Jul. 2004.

[4] F. Castells, J. J. Rieta, J. Millet, and V. Zarzoso, "Spatiotemporal blind source separation approach to atrial activity estimation in atrial tachyarrhythmias", IEEE Transactions on Biomedical Engineering, vol. 52, no. 2, pp. 258-267, Feb. 2005.
[5] V. Zarzoso, "Extraction of ECG characteristics using source separation techniques: exploiting statistical independence and beyond", Advanced Biosignal Processing, A. Naït-Ali, Ed. Berlin, Heidelberg: Springer Verlag, 2009, ch. 2, pp. 15-47.

[6] L. N. Ribeiro, A. R. Hidalgo-Muñoz, and V. Zarzoso, "Atrial signal extraction in atrial fibrillation electrocardiograms using a tensor decomposition approach", in Proc. EMBC-2015, 37th Annual International Conference of the IEEE Engineering in Medicine and Biology Society, Milan, Italy, Aug. 25-29, 2015, pp. 6987-6990.

[7] L. N. Ribeiro, A. R. Hidalgo-Muñoz, G. Favier, J. C. M. Mota, A. L. F. de Almeida, and V. Zarzoso, "A tensor decomposition approach to noninvasive atrial activity extraction in atrial fibrillation ECG", in Proc. EUSIPCO-2015, XXIII European Signal Processing Conference, Nice, France, Aug. 31-Sept. 4, 2015, pp. 2576-2580.

[8] L. N. Ribeiro, A. L. F. de Almeida, and V. Zarzoso, "Enhanced block term decomposition for atrial activity extraction in atrial fibrillation ECG", in Proc. SAM-2016, 9th IEEE Sensor Array and Multichannel Signal Processing Workshop, Rio de Janeiro, Brazil, July 10-13, 2016.

[9] V. Zarzoso, "Parameter estimation in block term decomposition for noninvasive atrial fibrillation analysis", in Proc. CAMSAP-2017, IEEE International Workshop on Computational Advances in Multi-Sensor Adaptive Processing, Curaçao, Dutch Antilles, Dec. 10-13, 2017.

[10] P. M. R. de Oliveira and V. Zarzoso, "Source analysis and selection using block term decomposition in atrial fibrillation", in Proc. LVA/ICA-2018, 14th International Conference on Latent Variable Analysis and Signal Separation, Guildford, United Kingdom, Jul. 2-6, 2018, pp. 46-56.

[11] P. M. R. de Oliveira and V. Zarzoso, "Block term decomposition analysis in long segments of atrial fibrillation ECGs", in Proc. SBrT-2018, XXXVI Simpósio Brasileiro de Telecomunicações e Processamento de Sinais, Campina Grande, Brazil, Sep. 16-19, 2018, pp. 578-582.

[12] V. Zarzoso and P. Comon, "Robust independent component analysis by iterative maximization of the kurtosis contrast with algebraic optimal step size", IEEE Transactions on Neural Networks, vol. 21, no. 2, pp. 248-261, 2010.

[13] I. Jolliffe, Principal Component Analysis, Wiley Online Library, 2005.

[14] L. De Lathauwer, "Blind separation of exponential polynomials and the decomposition of a tensor in rank- $\left(l_{r}, l_{r}, 1\right)$ terms", SIAM Journal on Matrix Analysis and Applications, vol. 32, no. 4, pp. 1451-1474, 2011.

[15] D. L. Boley, F. T. Luk, and D. Vandevoorde, "Vandermonde factorization of a Hankel matrix", in Proceedings of the Workshop on Scientific Computing, Hong Kong, March 1997.

[16] N. Vervliet, O. Debals, L. Sorber, M. Van Barel, and L. De Lathauwer, Tensorlab 3.0, Available online, Mar. 2016. URL: https://www.tensorlab.net/ 